\title{
The Jefferson Lab High Power THz User Facility
}

\author{
J. M. Klopf, A. Greer, J. Gubeli, G. R. Neil, M. Shinn, T. Siggins, D. Waldman and G.P. Williams \\ Jefferson Lab, Newport News, VA 23606, USA \\ gwyn@mailaps.org
}

A. Todd and V. Christina

AES, Inc. PO Box 7455, Princeton, NJ 08543, USA

O. Chubar

Synchrotron Soleil,, L'Orme des Merisiers, Saint-Aubin - BP 48, 91192 Gif-Sur-Yvette,France

\begin{abstract}
We describe here, a high power (100 Watt average, $10 \mathrm{MW}$ peak) broadband $\mathrm{THz}$ facility based on emission from sub-picosecond bunches of relativistic electrons and the beam transport system that delivers this beam in to a user laboratory.
\end{abstract}

\section{Introduction}

Jefferson Lab operates a broadband terahertz $(\mathrm{THz})$ facility, which delivers an average power of over 100 Watts of light into a user laboratory. The light is generated by sub-picosecond $135 \mathrm{pC}$ bunches of relativistic electrons as they traverse a magnetic compression chicane prior to entering the optical cavity of the Jefferson Lab Free Electron Laser (FEL)[1-3]. The repetition rate of the electron bunches, and hence that of the light pulses, can be as high as 75 $\mathrm{MHz}$ continuous. In practice the bunch lengths can be varied, and Fig. 1 shows the output power calculated for 3 different bunch lengths[4]. However, assuming that 100 Watts are produced for a bunch length of $1 \mathrm{ps,} \mathrm{with} \mathrm{a} \mathrm{duty}$ factor of 1 ps every 13 nanoseconds, the peak power is over $10 \mathrm{MW}$. Niche applications of this unique source are pump-probe dynamics, both in and out of equilibrium, and large-scale imaging.

\section{Detailed Description of the Optics of the Facility.}

Here we describe the design of the beamline which is designed to deliver into a user laboratory, an approximately $\mathrm{f} / 8$ beam that is about $2 \mathrm{~mm}$ vertical $\times 3 \mathrm{~mm}$ horizontal in size. Under the present configuration, the beam is $60 \%$ vertically polarized. The beamline extracts light emitted into a subtended angle of $\sim 200$ milliradians horizontal by $\sim 135$ milliradians vertical. The FEL is in an underground vault for shielding reasons, and the laboratories lie one floor above necessitating a long transport system. A further constraint on the optical design is the desire for a jog in the optics to allow radiation shielding to be installed that blocks line of sight between the FEL and the laboratory.

The philosophy of the optical design is to use a relay optics configuration to transport the beam via reflection off metal mirrors through a series of focal points. Thus the beam is focused at F1 onto a diamond window with unit magnification, then re-focused at an intermediate point F2, and finally re-focused at F3 at a second diamond window in the THz user laboratory. The final focus is a 1:1 image of the source. The difference in elevation between the electron beam and the beam delivery in the THz user lab is 5.8 meters.

The optical system is based on 6" optics and is shown schematically in Fig. 2. M1 is a 1:1 ellipsoidal mirror of focal length $625 \mathrm{~mm}$, which reflects the beam vertically upwards using s-polarization providing a focus at F1, roughly midway between M1 and M2. M2 and M4 are an identical pair of ellipsoids with focal lengths 705 and 2426 arranged such that M2 provides a source image at F2 magnified by 3.4, while M4 reduces this image by the same factor to give the 1:1 image on the diamond window at F3. F3 is 1 meter above the floor in the THz user lab. The intensity profile of the beam has been measured just behind the F3 diamond window using a Spiricon, Inc. Pyrocam. This is shown as well in Fig. 2. Mirror M3 which is not indicated on the figure is a plane mirror at the bottom of the very long vertical pipe.

The initial part of this beamline is in FEL machine vacuum of $10^{-9}$ torr. Mirror M1 is provided with a copper braid for cooling and is remotely controllable. The optical mount is a standard one with stepper motor driven screws. At both the F1 and F3 focus, there are $20 \mathrm{~mm}$ diameter 1 degree wedged diamond windows which provide vacuum integrity to either side. At F1, the window separates machine vacuum from the 100 millitorr vacuum of the remainder of the beamline, and at F3, the THz beam enters the lab either at atmosphere or vacuum, depending on the experimental configuration for the user. The M2 mirror is also remotely controllable, since it is in the accelerator 
vault, while M3 and M4 must be controlled manually, but can remain under vacuum. The full beamline design incorporates safety shutters, valves and cameras to view light images on insertable screens, plus alignment lasers to enable preliminary alignment to take place in the absence of beam.

The optical scheme was developed using the Synchrotron Radiation Workshop (SRW) code[5]. This code performs a full calculation of the electric field from a relativistic electron. It does not handle multiparticle coherent enhancement, but it is significantly different from all the other synchrotron radiation calculations because it retains a term called the Coulomb term in which the electric field falls off as $1 / \mathrm{R}^{2}$. In almost all cases of normal synchrotron radiation beamlines this is an unimportant term, but it is very important in the JLab case, making it essential to use this code. In addition to calculating the electric field and from this, the intensity, the code also allows one to propagate the field through the optical focusing elements and apertures.

The patterns of light at various locations in the transport system shown in Fig. 2 were obtained from the SRW code calculated at $1 \mathrm{THz}$. The circles indicate the approximate sizes of the apertures at the optical elements. The calculations were performed for a $100 \mathrm{MeV}$ beam. Note that the ring pattern originates from interference between the electric field generated by the magnetic field change on leaving one magnet and the one generated on entering the next magnet. The synchrotron radiation is manifest as the double increase in the center of the vertical stripes to the left of the figure. Taking account of the finite electron beam emittance made no discernible difference to the patterns, changing the electron beam energy from $80 \mathrm{MeV}$ to $150 \mathrm{MeV}$ made a discernible but unimportant difference. We also experimented with toroidal mirrors but there was no significant advantage and since we do not wish to change the anamorphic ratio, we retained ellipsoidal figures. Actually the differences between elliptical and toroidal figures are small.

\section{User Facility}

The beam exiting the final diamond window in the $\mathrm{THz}$ user lab passes into a beam conditioning vacuum chamber which allows attenuation, collimation, and/or beam splitting to take place for imaging, spectroscopy and pumpprobe measurements.

\section{Acknowledgements}

We deeply appreciate the excellent support of our colleagues without which these facilities would not have been built and operated. This work was supported by the U.S. Dept. of Energy under contract DE-AC05-84-ER40150, the Office of Naval Research, the Army Night Vision Laboratory, the Air Force Research Laboratory, the Commonwealth of Virginia and the Laser Processing Consortium.

\section{References}

1. G.R. Neil, C.L. Bohn, S.V. Benson, G. Biallas, D. Douglas, H.F. Dylla, R. Evans, J. Fugitt, A. Grippo, J. Gubeli, R. Hill, K. Jordan, G. Krafft, R. Li, L. Merminga, P. Piot, J. Preble, M. Shinn, T. Siggins, R. Walker and B. Yunn, "Sustained kilowatt lasing in a free-electron laser with same cell energy recovery" Physical Review Letters 84, 662 (2000).

2. G.L. Carr, M.C. Martin, W.R. McKinney, K. Jordan, G.R. Neil and G.P. Williams "High Power Terahertz Radiation from Relativistic Electrons", Nature 420 153-156 (2002).

3. G.P. Williams, "Far-IR/THz radiation from the Jefferson Lab energy recovered linac, free electron laser" Rev. Sci. Instr. 73 1461 (2002).

4. C. J. Hirschmugl, M. Sagurton and G. P. Williams, "Multiparticle coherence calculations for synchrotron radiation emission" Physical Review A44, 1316 (1991).

5. O. Chubar, P. Elleaume, "Accurate And Efficient Computation Of Synchrotron Radiation In The Near Field Region", proc. of the EPAC98 Conference, 22-26 June 1998, p.1177-1179. 


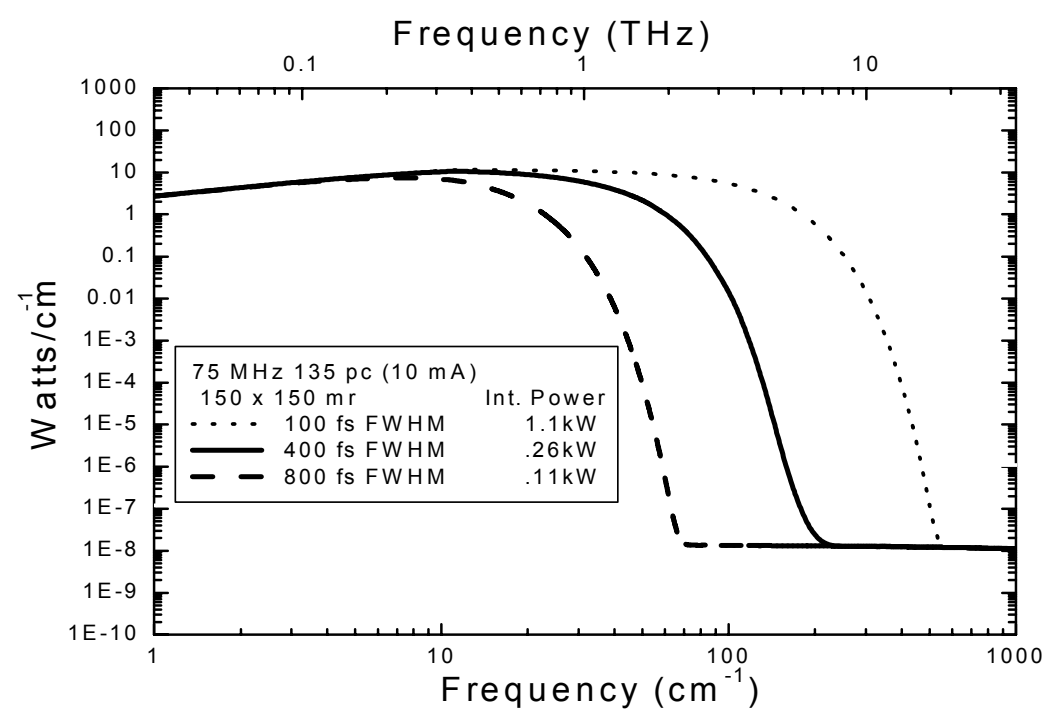

Fig. 1. Calculations of emitted power from the Jefferson Lab $\mathrm{THz}$ source for 3 different values of the electron bunch length all for an electron bunch charge of $135 \mathrm{pC}$ and 75 MHz repetition frequency 


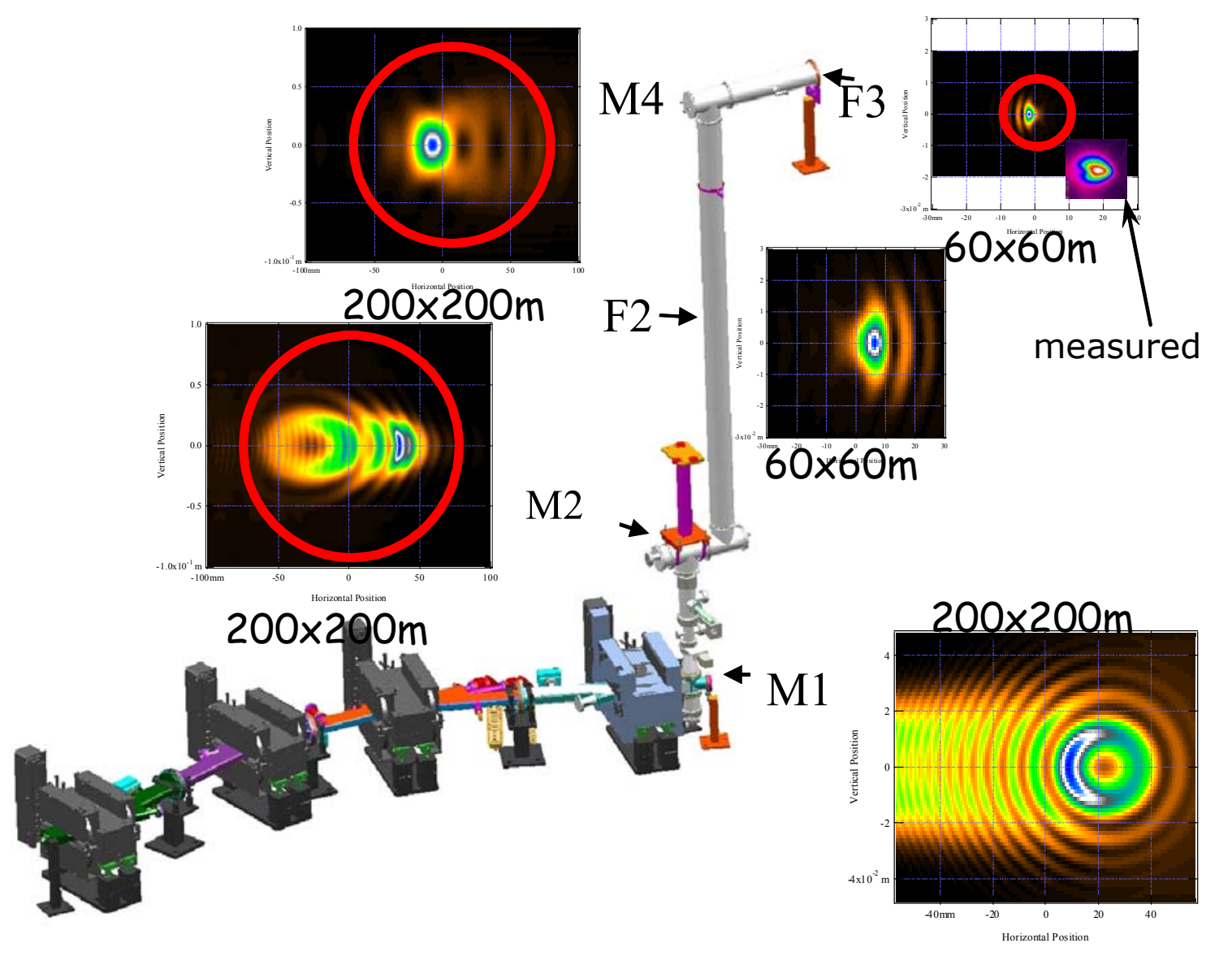

Fig. 2. 3-D Schematic of the Jefferson Lab THz beamline showing the FEL electron beam chicane at the bottom, and showing the radiation power patterns at indicated locations (spatial dimensions of each calculated power pattern shown in $\mathbf{m m}$ ). The red circles indicate the extent of the mirror or diamond window at that location, and the $\mathrm{THz}$ intensity measured at $\mathrm{F} 3$ is shown in the inset image. 